\title{
BEBIDA FERMENTADA A BASE DE ARROZ CON ADICIÓN DE PROBIÓTICOS
}

\author{
FERMENTED RICE-BASED DRINK WITH PROBIOTIC ADDITION
}

\author{
Ríos, P. Cindy, *Maldonado, M. Lida, Caballero, P., Luz.
}

\begin{abstract}
Universidad de Pamplona, Km. 1 Vía Bucaramanga, Pamplona, Colombia. Facultad de Ingenierías y Arquitectura. Programa de Ingeniería de Alimentos. Grupo de Investigaciones GIBA. Correo: * Iymaldonadom@gmail.com-
\end{abstract} luzcaballero@unipamplona.edu.co

Recibido 21 de Octubre 2015; aceptado 30 de Marzo de 2016

\section{RESUMEN}

En Colombia el masato es considerado una bebida tradicional, consumida como aperitivo y elaborada en forma artesanal, sin control de variables pues no existe una regulación específica para su control y vigilancia, se toman como parámetros los establecidos en el Decreto 1686 del 9 de agosto de 2012 que establece el Reglamento Técnico sobre los Requisitos Sanitarios que se deben cumplir para la fabricación, elaboración de bebidas alcohólicas, así como la Norma Técnica Colombiana 4092 y la Guía Técnica Colombiana GTC 4, Manual de métodos analíticos para el control de calidad de bebidas alcohólicas. El objetivo del trabajo fue elaborar una bebida fermentada a base de arroz con adición de probióticos con el fin de estandarizar el proceso artesanal de elaboración e incluir un valor agregado al consumidor. Para el proceso de elaboración se utilizaron diferentes concentraciones de azúcar 
Autor a quien dirigirse la correspondencia. Maldonado, M. Lida. Correo electrónico: lymaldonadom@gmail.com
(10\%, $15 \%, 20 \%$ ) para tres formulaciones, controlando el tiempo de fermentación (alcohólica) hasta obtener un grado de alcohol de1,5\% volumen (grado alcohol masato comercial); se realizaron pruebas químicas $(\mathrm{pH}$, sólidos solubles, acidez y grado de alcohol), sensoriales y microbiológicas con el fin de determinar la mejor formulación y tratamiento (pasteurizado y sin pasteurización). A la formulación seleccionada y pasteurizada se le adicionó un cultivo probiótico (Lactobacillus acidophilus), posteriormente se realizó análisis químico, microbiológico y sensorial, determinando la viabilidad de los microorganismos durante 12 días de almacenamiento en refrigeración $\left(4+/-2{ }^{\circ} \mathrm{C}\right)$. Se determinaron las diferencias mínimas significativas entre tratamientos. La bebida seleccionada fue la concentración de 20 \% de azúcar y un tiempo de fermentación de 12 horas. Se encontró que a los 12 días de almacenamiento en la bebida fermentada prevalecían $2 \times 10^{6} \mathrm{ufc} / \mathrm{ml}$ de cepas de Lactobacillus, con buena aceptación sensorial por parte de los jueces.

Palabras clave: bebida autóctona, calidad, fermentación alcohólica, masato, probióticos.

\section{ABSTRACT}

In Colombia the masato is considered a traditional drink, consumed as an appetizer and elaborated in a traditional way, without any control of variables since there is no specific regulation for its control and surveillance; the parameters taken are those established in Decree 1686 of August 9th, 2012 that establishes the Technical Regulation on the Sanitary Requirements that must be complied for the manufacture, preparation of alcoholic beverages, the Colombian Technical Standard 4092 and the Colombian Technical Guide GTC 4, 
Manual of analytical methods for the control of the quality of alcoholic beverages. The objective of the work was to elaborate a fermented rice-based drink with probiotic addition in order to standardize the process of elaboration and to include a value-added. For the process of elaboration different concentrations of sugar (10\%, $15 \%, 20 \%)$ were used for the three formulations, controlling the time of fermentation (alcoholic) until obtaining a degree of alcohol of $1.5 \%$ volume (alcohol grade of commercial masato); chemical $(\mathrm{pH}$, soluble solids, acidity and alcohol degree), sensorial and microbiological tests were carried out in order to determine the best formulation and treatment (unpasteurized and pasteurized). A probiotic culture (Lactobacillus acidophilus) was added to the selected formulation, followed by a chemical, microbiological and sensory analysis, determining the viability of the microorganisms during 12 days of refrigeration storage $\left(4+/-2{ }^{\circ} \mathrm{C}\right)$. Minimum significant differences between treatments were determined. The selected beverage was the one with a concentration of $20 \%$ sugar and a fermentation time of 12 hours. It was found that at 12 days of storage in the fermented beverage $2 \times 10^{6} \mathrm{cfu} / \mathrm{mL}$ of Lactobacillus strains prevailed, with a good sensorial acceptance by the judges.

Key words: traditional drink, alcoholic fermentation, masato, probiotics.

\section{INTRODUCCIÓN}

El masato es una bebida tradicional colombiana, preparada con maíz, arroz o una mezcla de los dos, con características muy propias como la textura, la cantidad de alcohol y el sabor dulce (Fula, 2010). En la elaboración de productos autóctonos la problemática más relevante es la falta de industrialización de dichos productos siguiendo parámetros sanitarios. Por su elaboración artesanal se utilizan cultivos 
iniciadores (guarapos) que no han sido tratados adecuadamente y pueden contener, además de las levaduras fermentativas, otro tipo de microorganismos contaminantes.

Según Guevara et al., (2005), en años recientes se presenta un creciente interés por agentes probióticos que contribuyan al equilibrio microbiano intestinal del consumidor, permitiendo controlar microorganismos patógenos por medio de la estimulación del sistema inmune, acidificando el contenido intestinal y aportando bacterias benéficas, además de las levaduras que aportan vitaminas $y$ enzimas bacterianas que colaboran en una mejor degradación del alimento consumido. La industria de bebidas con probióticas ha tenido un crecimiento exponencial, gracias a que la tendencia en estas últimas décadas de una alimentación saludable, por su

comprobado efecto positivo en la calidad de vida de las personas que las consumen (Fula, 2010).

El presente trabajo tuvo como objetivo la elaboración de una bebida a base de arroz denominada "masato", fermentada a partir de levadura (Saccharomyces cerevisiae), estandarizando el proceso de elaboración e identificando al cabo de 12 días de almacenamiento en refrigeración $\left(4 \pm 2{ }^{\circ} \mathrm{C}\right)$, la viabilidad de la adición del cultivo probiótico (Lactobacillus acidophilus) mediante análisis químicos, sensoriales y microbiológicos. Actualmente Colombia no cuenta con una normativa específica para este tipo de bebida por lo que se tuvo en cuenta la normatividad para bebidas alcohólicas.

\section{MATERIALES Y MÉTODOS}

Para llevar a cabo la elaboración del masato fue necesario determinar el grado de alcohol de una muestra comercial con el fin de establecer un parámetro de referencia, debido a la falta de fuentes bibliográficas para este tipo de bebidas. Durante la elaboración preliminar de la bebida se controló el proceso de fermentación hasta obtener un grado de alcohol similar a la muestra comercial.

Formulaciones preliminares. Para determinar las condiciones de fermentación del masato se desarrollaron tres formulaciones preliminares de elaboración de masato a base de recetas artesanales de diferentes regiones del país, siguiendo la 
metodología de Fula (2010). En la tabla 1 se presentan las formulaciones trabajadas variando la concentración de azúcar, manteniendo constantes la levadura y demás materias primas empleadas en la elaboración de 5 litros de masato.

Tabla 1. Formulaciones preliminares de la bebida fermentada a base de arroz. Base de Cálculo: 5 Litros.

\begin{tabular}{|l|c|c|c|}
\hline $\begin{array}{l}\text { Materia } \\
\text { prima }\end{array}$ & $\begin{array}{c}\text { Formulació } \\
\mathbf{n} \mathbf{1}\end{array}$ & $\begin{array}{c}\text { Formulació } \\
\mathbf{n ~ 2}\end{array}$ & $\begin{array}{c}\text { Formulació } \\
\mathbf{n ~ 3}\end{array}$ \\
\hline Arroz & $10 \%$ & $10 \%$ & $10 \%$ \\
\hline Agua & $79,3 \%$ & $73,8 \%$ & $68,3 \%$ \\
\hline Azúcar & $10 \%$ & $15 \%$ & $20 \%$ \\
\hline Levadura & $0,5 \%$ & $0,5 \%$ & $0,5 \%$ \\
\hline $\begin{array}{l}\text { Sorbato } \\
\text { de } \\
\text { Potasio }\end{array}$ & $0,5 \mathrm{mg} / \mathrm{L}$ & $0,5 \mathrm{mg} / \mathrm{L}$ & $0,5 \mathrm{mg} / \mathrm{L}$ \\
\hline $\begin{array}{l}\text { Extracto } \\
\text { de clavos } \\
\text { y canela }\end{array}$ & $0,2 \%$ & $0,2 \%$ & $0,2 \%$ \\
\hline
\end{tabular}

La levadura utilizada en el proceso de fermentación fue la Saccharomyces cerevisiae, seca, la cual se activó tomando $100 \mathrm{ml}$ de agua a $35-40{ }^{\circ} \mathrm{C}$, adicionando 2 $\mathrm{g}$ de azúcar y $5 \mathrm{~g}$ de levadura; se dejó en reposo por media hora controlando la temperatura por medio de una plancha de calentamiento.

En la figura 1 se describen las etapas definidas para la elaboración del masato y las variables de control adaptadas partir de la metodología propuesta por Fula (2010) y Guerrero (2011).

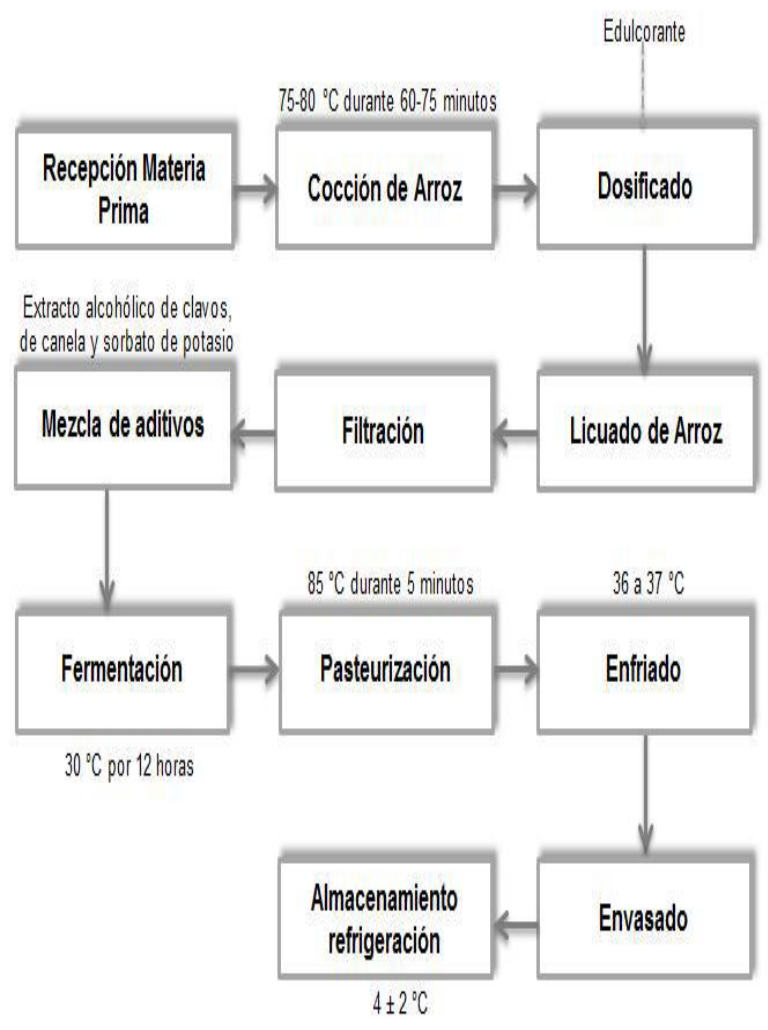

Figura 1. Procedimiento de elaboración de masato a partir de arroz adaptado por los autores.

Durante la elaboración de la bebida se controló la etapa de fermentación midiendo las siguientes características cada tres horas: $\mathrm{pH}$, acidez, sólidos solubles y grado de alcohol hasta alcanzar el grado de alcohol determinado en la bebida comercial, seleccionando así la formulación que alcanzo las mejores características evaluadas. 
Las formulaciones de las bebidas fermentadas a partir de arroz (5 Litros), se dividieron: 2,5 litros se pasteurizaron a $85 \pm$ $1{ }^{\circ} \mathrm{C}$ por 5 minutos y la otra mitad se dejó sin pasteurizar, obteniendo dos tratamientos (bebida fermentada pasteurizada y sin pasteurizar). Ambos tratamientos fueron evaluados y analizados durante 12 días de almacenamiento en refrigeración $\left(4 \pm 2^{\circ} \mathrm{C}\right)$. Las características químicas se evaluaron según los parámetros establecidos en la GTC 4 los días 3, 6, 10 y 12 de almacenamiento en refrigeración $\left(4 \pm 2{ }^{\circ} \mathrm{C}\right)$. $\mathrm{El} \mathrm{pH}$ se evaluó mediante un $\mathrm{pH}$-metro Mettler TOLEDO. La determinación de grados ${ }^{\circ}$ Brix se midió con un refractómetro digital REICHERT AR 200, calibrado a $20 \pm$ $2{ }^{\circ} \mathrm{C}$, registrándose la lectura en escala de 'Brix. La determinación de grados de alcohol se realizó por destilación simple conforme a la metodología establecida por la GTC 4. EI porcentaje de alcohol se determinó mediante la tabla de porcentaje de alcohol a 15,56 \pm 1 ${ }^{\circ} \mathrm{C}$ correspondientes a la gravedad específica a temperatura $20 / 20 \pm 1{ }^{\circ} \mathrm{C}$.

Gravedad específica a $\underline{20}{ }^{\circ} \mathrm{C}=\underline{\mathrm{Pc}-\mathrm{Po}}$

$$
\mathrm{Pa}-\mathrm{Po}
$$

$\mathrm{Pc}=$ peso del picnómetro con muestra, en $\mathrm{g}$

$\mathrm{Pa}=$ peso del picnómetro con agua en, $\mathrm{g}$

$\mathrm{Po}=$ peso del picnómetro vacío, en $\mathrm{g}$

(Ecuación 1)

La acidez se determinó por el método descrito en la GTC 4 de 1994 como porcentaje de ácido láctico. Para ello se tomaron $200 \mathrm{ml}$ de agua destilada a $100 \pm 2$ C y se mezcló con $25 \mathrm{ml}$ de muestra de la bebida, titulando posteriormente con $\mathrm{NaOH}$ $0,1 \mathrm{~N}$ hasta alcanzar un $\mathrm{pH}$ al 8,2 a $20^{\circ} \mathrm{C}$. $\mathrm{La}$ acidez se calculó mediante la siguiente ecuación:

Acidez total $=\mathrm{cm}^{3}$ de $\mathrm{NaOH} 0.1 \mathrm{~N} * 0.9 / \mathrm{cm} 3$ de masato por la gravedad especifica de masato_(Ecuación 2)

$0.09=\mathrm{cm}^{3}$ equivalentes de una solución de ácido láctico $1 \mathrm{~N}$.

Una vez pasteurizado el masato, se evaluó microbiológicamente en el Laboratorio de Control de Calidad y Diagnostico de la Universidad de Pamplona. Se realizó recuento total de microorganismos mesofilos y recuento total de mohos y levaduras, (NTC 3854 de 1996).

Se realizó una prueba sensorial de aceptación a la bebida después del proceso de fermentación con el fin de determinar la mejor formulación. La misma prueba se repitió a los 12 días de almacenamiento para identificar el mejor tratamiento (bebida pasteurizada y no pasteurizada). Los resultados se estimaron con un panel de 36 consumidores habituales del producto y las muestras se presentaron en series diciendo 
a los catadores que se trata del mismo productos con diferente tratamiento, (Sancho, Bota y Castro, 1999).

A partir de los resultados obtenidos se determinó la mejor formulación y tratamiento (pasteurizado y sin pasteurización).

\section{Adición de microorganismos probióticos la bebida fermentada a base de arroz seleccionada.}

A la formulación pasteurizada seleccionada se le adiciono un cultivo probiótico (Lactobacillus acidophilus) realizándole un análisis químico, microbiológico y sensorial, determinando la viabilidad de los microorganismos durante 12 días de almacenamiento en refrigeración $\left(4+/-2^{\circ} \mathrm{C}\right)$. Se inoculo el cultivo probiótico (Lactobacillus acidophilus), siguiendo los parámetros de calidad necesarios para evitar la contaminación, la cantidad suministrada se calculó a partir de la relación en la ficha técnica, la cual correspondía a 24,3 g para $1200 \mathrm{~L}$ de producto, obteniéndose que para $5 \mathrm{~L}$ eran necesarios adicionar $0,10125 \mathrm{~g}$. La incubación de la bebida se llevó a cabo a temperatura de $37 \pm 1^{\circ} \mathrm{C}$, según lo estimado por la ficha técnica suministrada por la empresa CIMPA S.A.S., Insumos y tecnología para la industria alimentaria.

Análisis químico. El periodo de incubación fue controlado evitando diferencias significativas respecto a los análisis químicos como $\mathrm{pH}$, acidez, sólidos solubles y grado de alcohol, determinados en la estandarización de la bebida fermentada a base de arroz.

Análisis microbiológico y viabilidad. Se realizaron conteos microbiológicos de las bacterias probióticas inoculadas. Para este análisis se utilizó la metodología descrita en la NTC 4092 de 2009. El conteo de bacterias probióticas se realizó los días 0, 5 y 12 de almacenamiento en refrigeración $\left(4 \pm 1^{\circ} \mathrm{C}\right)$. Las siembras se realizaron por duplicado en diferentes diluciones. Para el análisis microbiológico se realizó siembra en placa y en profundidad de las muestras. En agar M.R.S. (Man, Rogosa y Sharpe) fundido, se homogenizo y se incubo a $32-37 \pm 1 \stackrel{\circ}{\circ}$ en aerobiosis durante un periodo de 24 - $48 \mathrm{~h}$, al cabo de las cuales se efectuó el conteo de las colonias formadas.

Evaluación sensorial. Se aplicó una prueba afectiva de la bebida fermentada a base de arroz con adición de probióticos en los días 10 y 12 de almacenamiento, con el fin de determinar su aceptación. Los resultados se estimaron con un panel de consumidores habituales cuyo número fue de 36 , las muestras se presentaron en series de 15 , se pide que los prueben y manifiesten si se trata del original o de un producto distinto. 
Análisis estadístico de resultados. Los resultados obtenidos en las diferentes actividades se analizaron estadísticamente empleando el paquete SPSS versión 13, aplicándose el análisis de varianza (ANOVA), asumiendo un nivel de significancia del $95 \%$ con el fin de establecer la formulación adecuada para la estandarización del masato y definir el tiempo de almacenamiento de la bebida inoculada con microrganismos probióticos.

Se empleó la prueba de comparación múltiple POST-HOC para determinar diferencias mínimas significativas entre tratamientos de las variables de respuesta estudiadas $(\mathrm{pH}$, solidos solubles, acidez y grado de alcohol).

\section{RESULTADOS Y DISCUSIÓN}

El grado de alcohol obtenido de la muestra comercial de masato adquirida en el municipio de Pamplona, Norte de Santander fue de $1,5 \%$ volumen de alcohol. Durante el proceso de fermentación de la bebida autóctona a base de arroz se toma de referencia este valor como óptimo. En la elaboración de la bebida fermentada la cocción del arroz presento un tiempo de 60 75 minutos a temperatura de $75-80 \pm 1 \stackrel{\circ}{\circ}$, para la dilución del azúcar se requirió una temperatura de $70 \pm 1 \stackrel{\circ}{\circ}$. Para otros productos amiláceos la temperatura de cocción es variable, en el caso de la preparación del saque, a partir de arroz, (como se cita en Sotero et al., 1996), afirman que el tiempo de cocción es de 60 minutos con vapor. La fermentación fue interrumpida después de 12 horas de incubación a $35 \pm 1$
${ }^{\circ} \mathrm{C}$, tiempo en el que se alcanzó la concentración de alcohol deseado (1,5\% volumen de alcohol), según la muestra comercial tomada como referencia.

A continuación se presentan los resultados obtenidos luego de realizar la fermentación a $35 \pm 1 \quad \stackrel{\circ}{C}$ en incubadora de tres formulaciones con concentraciones de azúcar de 10,15 y $20 \%$ y concentraciones de levadura al 0,1 \% para todas las formulaciones.

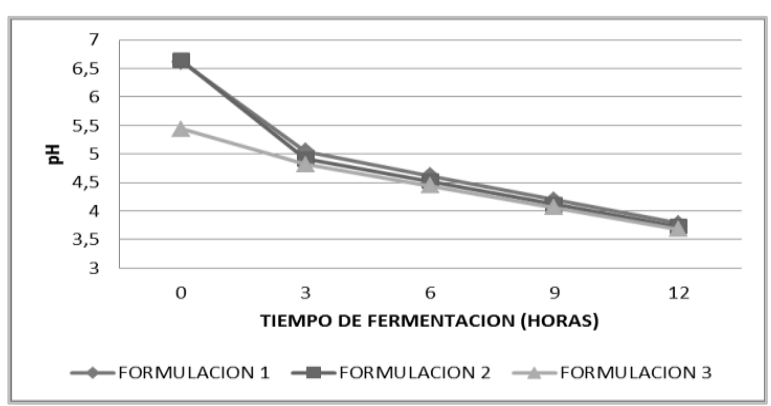


@ LIMENTECH CIENCIA Y TECNOLOGÍA ALIMENTARIA ISSN 1692-7125. Volumen 14 No. 1, p. 58 - 73, año 2016 Facultad de Ingenierías y Arquitectura Universidad de Pamplona

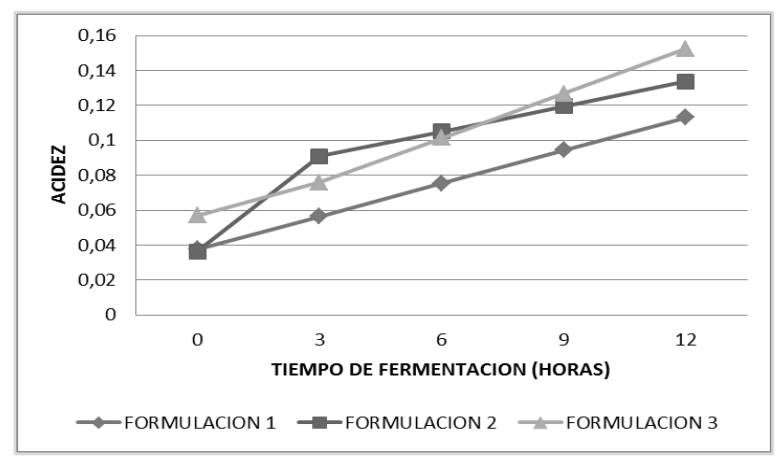

Figura 2. Evolución del $\mathrm{pH}$ y la acidez durante la fermentación de la bebida a base de arroz.

De la figura 2 en cuanto a los resultados de la variación del pH durante la fermentación para las tres formulaciones $(10,15$ y $20 \%$ de azúcar), se observa que el día 0 la formulación 1 y 2 presentó $\mathrm{pH}$ similares $(6,62$ y 6,64$)$ y al cabo de las 12 horas se evidencio que en las tres formulaciones disminuyó, alcanzando un $\mathrm{pH}$ similar $(3,78,3,73,3,68$ respectivamente). Se recomiendan para este tipo de bebidas $\mathrm{pH}$ menores que 4, por cuanto así presenta esta una mejor resistencia a la infección bacteriana (Sotero et al., 1996).

La acidez aumentó con el paso de las horas, la formulación 2 incremento después de tres horas $(0,03$ - 0,09\%), por otra parte la formulación 1 y 3 mostraron aumento similar aunque la formulación 3 (20 \% azúcar) fue la que mayor acidez alcanzo $(0.15 \%)$ cuando se interrumpió la fermentación por el grado de alcohol requerido.
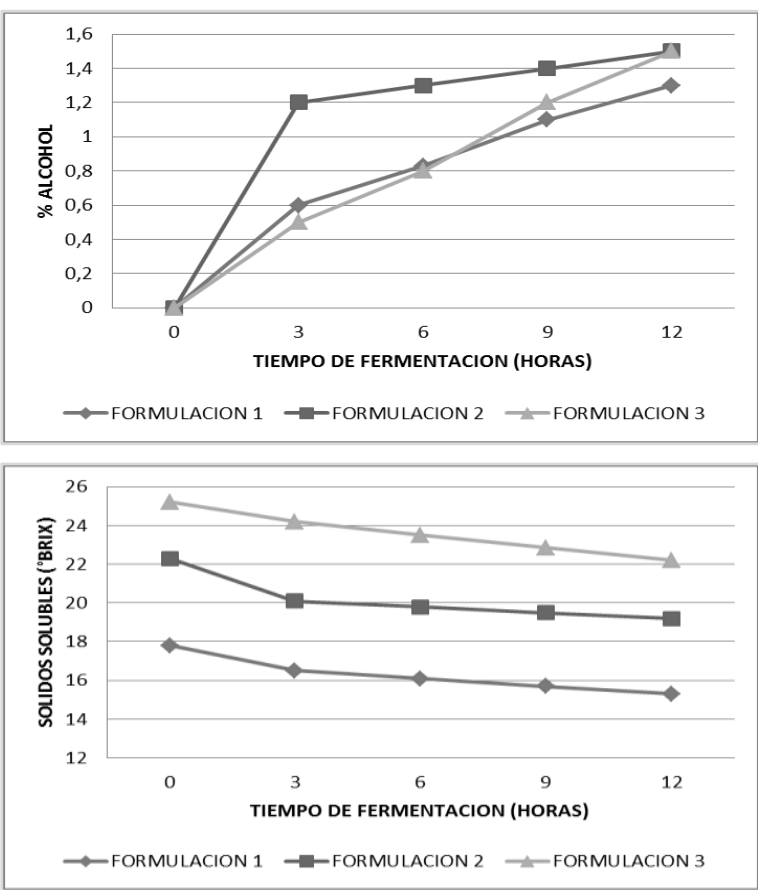

Figura 3. Evolución del \% de alcohol y los sólidos solubles ( ${ }^{\circ}$ Brix) durante la fermentación de la bebida a base de arroz.

El consumo de azucares por la levadura Saccharomyces cerevisiae durante la fermentación se observa en la figura 3 , la formulación 3 presento un elevado valor de sólidos solubles (25,3 ${ }^{\circ}$ Brix) al inicio de la fermentación, posiblemente debido a que los azucares presentes en la masa de arroz fueron desdoblados durante el proceso de cocción, a las tres horas de fermentación los sólidos solubles comienzan a decrecer para las tres formulaciones, evidenciándose disminución de los Brix los cuales están relacionados con el grado de alcohol.

Durante los primeros días del proceso de fermentación, las células de levadura crecen 
por respiración consumiendo oxígeno y (20\% azúcar) para los atributos sensoriales creando un medio anaerobio. Tan pronto como el oxígeno desaparece se inicia la fermentación, produciendo alcohol, por tanto la levadura pasa de metabolismo aerobio al anaerobio (Ramírez y Pedroza, 2001).

En la evolución del grado de alcohol se evidencia que la formulación 2 a las 3 horas de fermentación incremento de 0 - 1,2\% volumen, mientras que la 1 y 3 aumentaron más lentamente de $0-0,6 \%$ y de 0 - 0,5\% volumen, luego de 12 horas de fermentación la formulación 2 y 3 alcanzaron el grado de alcohol requerido (1,5\% volumen), por lo cual se detuvo la fermentación evitando el incremento del contenido de alcohol y por consiguiente se generaron cambios sensoriales en la bebida principalmente en el sabor y olor.

Además de las características químicas evaluadas para la determinación de la mejor formulación fue necesario realizar una evaluación sensorial ya que la formulación 2 y 3 (15 y $20 \%$ azúcar respectivamente) presentaron parámetros químicos similares, obteniendo grado de alcohol igual (1,5\% volumen).

En la prueba sensorial aplicada a las tres formulaciones luego de la fermentación, se obtuvo mayor aceptación de la formulación 3

\begin{tabular}{|l|c|c|}
\hline $\begin{array}{c}\text { FORMULACION } \\
\mathbf{3}\end{array}$ & $\begin{array}{c}\text { INICIAL } \\
\text { (0 horas) }\end{array}$ & $\begin{array}{c}\text { FINAL } \\
\text { (12 horas) }\end{array}$ \\
\hline pH & 5,44 & 3,68 \\
\hline Acidez & 0,05 & 0,15 \\
\hline${ }^{\circ}$ Brix & 25,2 & 22,2 \\
\hline Alcohol & 0 & 1,5 \\
\hline
\end{tabular}

La Tabla representa los resultados evolutivos que describen el comportamiento del pH en el almacenamiento de la bebida a base de arroz, en la muestra sin pasteurizar se observa un descenso que se relaciona directamente con el incremento de la acidez, de 3,68 a 3,06 en los 12 días de almacenamiento, la muestra pasteurizada se mantuvo constante a partir del 6 día, con pH final de 3,6. 
$\mathrm{El} \mathrm{pH}$ es una característica de primaria importancia, esto por diferentes motivos ante todo organolépticamente, el $\mathrm{pH}$ influye fuertemente sobre la sensación de acidez, dado que esta depende más de la concentración hidrogenionica (fuerza ácida) que de la cantidad de ácidos contenidos (Prieto et al., 2001).

Por otra parte se observa que la muestra pasteurizada disminuyo lentamente el porcentaje de acidez aunque luego del décimo día fue constante manteniendo la acidez en 0,15 mg. Según Fula (2010) en investigaciones de bebidas pasteurizadas, esta tiene efecto sobre la acidez, debido a la evaporación de los compuestos volátiles, de tal manera que se reduce, además se inhibe la actividad de algunas enzimas causantes de la degradación de los azucares para su conversión en ácidos.

Se evidencia que la muestra pasteurizada presento un leve aumento de los sólidos solubles $(22,2-23,5){ }^{\circ}$ Brix, se observa que a partir del décimo día de almacenamiento se mantienen constantes, posiblemente la pasteurización tiene efecto sobre los sólidos solubles, ya que al realizar una pasteurización prolongada, se produce evaporación y por consiguiente aumento de los sólidos (Fula, 2010).
En la muestra no pasteurizada los sólidos solubles disminuyeron notablemente durante el almacenamiento finalizando con $20,2^{\circ}$ Brix indicando que la refrigeración no presenta efecto inhibidor de la levadura Saccharomyces cerevisiae puesto que esta continua el proceso de fermentación y por consiguiente aumenta el grado de alcohol 1,5 - 2,5 en comparación con la muestra pasteurizada la cual mantuvo estable el grado de alcohol en 1,5\%.

Visualmente se percibió que la producción de dióxido de carbono en la muestra no pasteurizada provoco hinchamiento del envase, en comparación con la muestra sometida a pasteurización que no presento producción de gas (CO2).

En la siguiente tabla se presentan los recuentos obtenidos de aerobios mesófilos, mohos y levaduras para la bebida fermentada a base de arroz luego del proceso de pasteurización.

Tabla 3. Resultados microbiológicos de la bebida fermentada a base de arroz.

\begin{tabular}{|c|c|c|}
\hline PARAMETRO & $\begin{array}{l}\text { Muestra } \\
\text { (masato) }\end{array}$ & NTC 3854 \\
\hline $\begin{array}{l}\text { Recuento total de } \\
\text { microorganismos } \\
\text { mesófilos }\end{array}$ & $\begin{array}{l}3 \times 10 \\
\text { UFC } / \mathrm{cm}^{3}\end{array}$ & $\begin{array}{l}\leq 100 \text { UFC } \\
/ \mathrm{cm}^{3}\end{array}$ \\
\hline $\begin{array}{l}\text { Recuento total } \\
\text { de mohos y } \\
\text { levaduras }\end{array}$ & $\begin{array}{lll}7 & X \quad 10 \\
\text { UFC/ } & \mathrm{cm}^{3}\end{array}$ & $\begin{array}{c}\leq 20 \underset{/ \mathrm{cm}^{3}}{\mathrm{UFC}} \\
\text {. }\end{array}$ \\
\hline
\end{tabular}


El recuento de aerobios mesófilos obtenidos indica que las muestras analizadas de la bebida fermentada a base de arroz, se encontraban dentro del límite establecido por la NTC 3854.

Las características fisicoquímicas de la bebida fermentada a base de arroz almacenada en refrigeración durante 12 días presentaron diferencias significativas.

Presentando mayor estabilidad la muestra sometida al proceso de pasteurización, ya que la no pasteurizada aumento el grado de alcohol y se disminuyeron los sólidos solubles debido a la presencia de la levadura Saccharomyces cerevisiae la cual continuo lentamente con el proceso de fermentación durante los 12 días de almacenamiento. Así mismo disminución del $\mathrm{pH}$ el cual influyo notablemente en la sensación de acidez luego del décimo día de almacenamiento.

Tabla 4. Resultados estadísticos de las características fisicoquímicas de la bebida fermentada a base de arroz.

\begin{tabular}{|c|c|c|c|c|}
\hline $\begin{array}{l}\text { TRATA } \\
\text { MIENTO }\end{array}$ & $\mathrm{pH}$ & $\mathrm{SS}\left({ }^{\circ} \mathrm{Bx}\right)$ & $\begin{array}{c}\% \\
\text { Alcohol }\end{array}$ & $\begin{array}{c}\mathrm{cm}^{3} \\
\text { Acidez }\end{array}$ \\
\hline Inicial & $\begin{array}{l}3,68 \pm \\
0,003 \text { a }\end{array}$ & $\begin{array}{l}22,20 \pm \\
0,000 \mathrm{c}\end{array}$ & $\begin{array}{l}1,5 \pm \\
0,000 \mathrm{~d}\end{array}$ & $\begin{array}{l}0,0600 \pm \\
0,000 \mathrm{f}\end{array}$ \\
\hline $\begin{array}{l}\text { Bebida } \\
\text { pasteuri } \\
\text { zada }\end{array}$ & $\begin{array}{l}3,62 \pm \\
0,010 \mathbf{b}\end{array}$ & $\begin{array}{l}23,39 \pm \\
0,062 \text { c }\end{array}$ & $\begin{array}{l}1,5 \pm \\
0,000 \mathrm{e}\end{array}$ & $\begin{array}{l}0,0428 \pm \\
0,002 \mathbf{g}\end{array}$ \\
\hline \begin{tabular}{l} 
Bebida \\
no \\
pasteuri \\
\multicolumn{2}{c}{ zada }
\end{tabular} & $\begin{array}{l}3,19 \pm \\
0,040 \text { a } \\
\text { b }\end{array}$ & $\begin{array}{l}20,60 \pm \\
0,119 \mathrm{c}\end{array}$ & $\begin{array}{l}2,3 \pm \\
0,062 \mathbf{d} \\
\mathbf{e}\end{array}$ & $\begin{array}{l}0,1909 \pm \\
0,018 \mathbf{f} \mathbf{g}\end{array}$ \\
\hline$p$-valor & 0,000 & 0,000 & 0,000 & 0,000 \\
\hline
\end{tabular}

Media \pm desviación típica $p$-valor $\leq 0,05$ existen diferencias significativas del $95 \%$, a, $b, c, d, e, f, g$ letras iguales entre columnas existen diferencias mínimas significativas.

Mediante la prueba sensorial de aceptación se determina el proceso de pasteurización como el mejor tratamiento presentando olor y sabor agradable, acidez suficiente, textura buena con un $100 \%$ de aceptabilidad.

La incubación del cultivo probiótico fue interrumpida luego de 48 horas debido a los cambios químicos en cuanto al pH, acidez, solidos solubles y grado de alcohol con respecto a la bebida fermentada a base de arroz estandarizada, como se muestra a continuación.
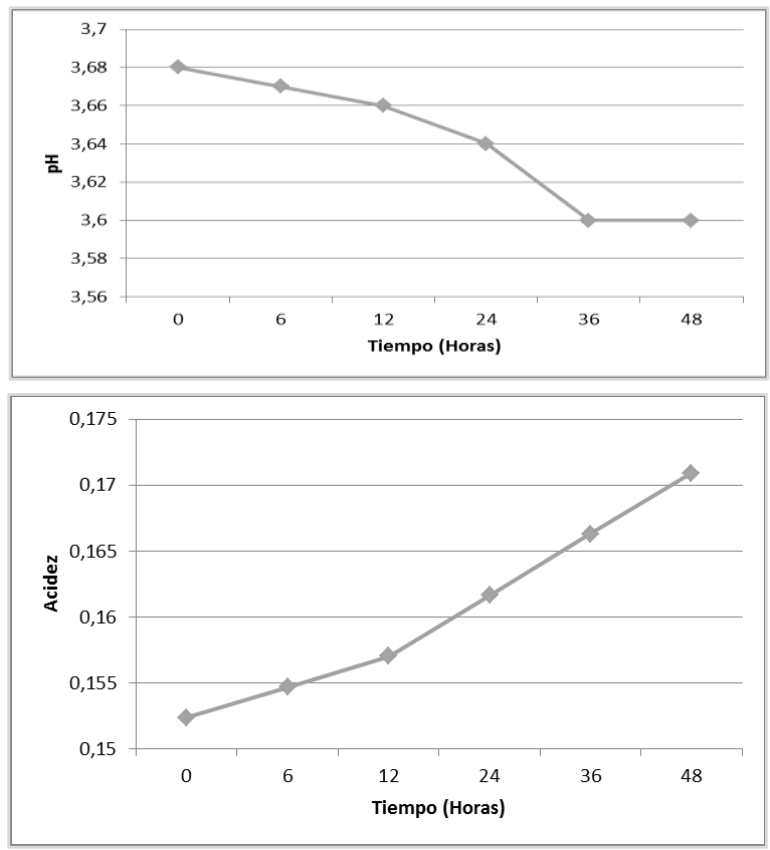
Figura 4. Evolución del $\mathrm{pH}$ y acidez en la incubación del probiótico.

El aumento de la acidez representa a nivel sensorial, acidificación de la bebida siendo este un parámetro característico dado que se relaciona con el desarrollo del flavor, por tanto un nivel de acidez muy bajo deja al producto final con un sabor no característico de un masato (medianamente acido).

Las bacterias lácticas transforman los azucares en metabolitos de interés industrial como etanol y ácidos orgánicos. Muchas especies del genero Lactobacillus tienen la capacidad de competir en ambientes fermentativos por su alto nivel de tolerancia al etanol (Bautista, 2006), Los sólidos solubles se disminuyeron con la presencia del probiótico durante la incubación alcanzando 18,9 ${ }^{\circ}$ Brix, indicando consumo de azucares por parte de la bacteria, así mismo se evidencia aumento del grado de alcohol a partir de la 6 hora de incubación alcanzando 1,8 \% volumen de alcohol presentando cambio en 0,3\% volumen de alcohol con respecto a la bebida fermentada y pasteurizada con $1,5 \%$ volumen de alcohol, por tanto se hace necesario realizar estudios que permitan determinar la causa del incremento del contenido de alcohol. Las cepas de Lactobacillus muestran un alto nivel de tolerancia a concentraciones de etanol, hasta $12 \%$ v/v de etanol (Bautista, 2006).
Según el reporte de la FAO en el 2001 con respecto a la cantidad necesaria que debe contener una bebida para considerarse beneficiosa por la presencia de bacterias probióticos es de $>1 \times 10^{6}$ ufc, de tal manera que en la bebida fermentada elaborada a base de arroz se evidencia presencia del cultivo probiótico hasta culminar los 12 días de almacenamiento

Contreras et al., 2007 determinaron la presencia de Lactobacillus spp, (34 x 106 $\mathrm{ufc} / \mathrm{mL}$ ) en pulque bebida tradicional Mexicana que se obtiene por la fermentación de la savia azucarada conocida como aguamiel obtenida a partir de diferentes especies de maguey con porcentaje de alcohol de 10,35 \% volumen, donde concluyen que el Lactobacillus spp podrían tener efecto benéfico sobre el sistema digestivo al ser consumido por vía oral.

Tabla 5 Resultados de los análisis microbiológicos de la bebida fermentada.

\begin{tabular}{|c|c|c|c|}
\hline PARAMETRO & DIA & $\begin{array}{l}\text { MUES- } \\
\text { TRA } \\
\text { (masato) }\end{array}$ & $\begin{array}{l}\text { FAO (2001)/ } \\
\text { INVIMA }\end{array}$ \\
\hline $\begin{array}{c}\text { Recuento de } \\
\text { Lactobacillus } \\
\text { spp }\end{array}$ & 0 & $\begin{array}{l}3 \times 106 \\
\mathrm{ufc} / \mathrm{ml}\end{array}$ & $>1 \times 106$ ufc \\
\hline $\begin{array}{l}\text { Recuento de } \\
\text { Lactobacillus } \\
\text { Spp }\end{array}$ & 5 & $\begin{array}{l}2 \times 106 \\
\mathrm{ufc} / \mathrm{ml}\end{array}$ & 06 ufc \\
\hline $\begin{array}{l}\text { Recuento de } \\
\text { Lactobacillus } \\
\text { Spp }\end{array}$ & 12 & $\begin{array}{l}2 \times 106 \\
\text { ufc/ml }\end{array}$ & $>1 \times 106$ ufc \\
\hline $\begin{array}{l}\text { Recuento } \\
\text { microorganis } \\
\text { mos } \\
\text { mesófilos }\end{array}$ & 12 & $\begin{array}{l}18 x \\
102 \\
\text { ufc/ml }\end{array}$ & $\begin{array}{c}2 \times 105-3 \times \\
105 u f c / m l\end{array}$ \\
\hline
\end{tabular}


@ LIMENTECH CIENCIA Y TECNOLOGÍA ALIMENTARIA ISSN 1692-7125. Volumen 14 No. 1, p. 58 - 73, año 2016 Facultad de Ingenierías y Arquitectura Universidad de Pamplona

\begin{tabular}{|l|l|l|c|}
\hline $\begin{array}{l}\text { Recuento } \\
\text { mohos y } \\
\text { levaduras }\end{array}$ & 12 & $\begin{array}{l}17 \mathrm{x} \\
102 \\
\text { ufc/ml }\end{array}$ & $\begin{array}{r}1000-2000 \\
\mathrm{ufc} / \mathrm{ml}\end{array}$ \\
\hline
\end{tabular}

Se observa que los recuentos estuvieron por debajo de lo establecido por la Resolución 11888 y el Decreto 1506 de 2014 MPS INVIMA, luego de 12 días de almacenamiento en refrigeración $\left(4 \pm 1^{\circ} \mathrm{C}\right)$.

En la tabla 6 se muestra los resultados para cada uno de los atributos evaluados en la prueba sensorial encontrando que la bebida tuvo una aceptación sensorial al 10 día, presentando característica agradable en cuanto al sabor, olor, y grado de acidez.

Tabla 6. Resultados de las características sensoriales de la bebida fermentada adicionada con probióticos.

\begin{tabular}{|l|c|c|}
\hline $\begin{array}{c}\text { CARACTERISTICAS } \\
\text { SENSORIALES } \\
\text { BEBIDA } \\
\text { FERMENTADA }\end{array}$ & $\mathbf{1 0}$ días & $\mathbf{1 2 \text { días }}$ \\
\hline Olor & Agradable & Característico \\
\hline Sabor & Agradable & $\begin{array}{c}\text { Poco } \\
\text { agradable }\end{array}$ \\
\hline Àcidez & Buena & Muy Àcido \\
\hline Textura & Buena & Buena \\
\hline $\begin{array}{l}\text { Aceptabili- } \\
\text { Dad }\end{array}$ & $\begin{array}{c}\text { Gusta } \\
\text { mucho }\end{array}$ & $\begin{array}{c}\text { Gusta } \\
\text { levemente }\end{array}$ \\
\hline Olor & Agradable & Característico \\
\hline
\end{tabular}

Se logró establecer que el tiempo de almacenamiento en refrigeración óptimo para la bebida fermentada a base de arroz es de 10 días, ya que se observó al día 12 un significativo aumento de la acidez causando sabor desagradable a la bebida y menor aceptabilidad al consumidor.

\section{CONCLUSIONES}

Se logró estandarizar las concentraciones de materias primas para elaborar una bebida fermentada elaborada a base de arroz con $20 \%$ de azúcar, controlando variables como tiempo (12 horas) y temperatura $\left(35 \pm 1^{\circ} \mathrm{C}\right)$ durante la etapa de fermentación.

Se determinaron las características químicas de la bebida fermentada elaborada a base de arroz en almacenamiento durante 12 días obteniendo $\mathrm{pH}$ de 3,68 , solidos solubles de $22,02{ }^{\circ}$ Brix y grado de alcohol de $1,5 \%$.
Sensorialmente la bebida fermentada y pasteurizada con concentración de $20 \%$ de azúcar obtuvo el $100 \%$ de aceptabilidad por parte de los jueces evaluadores.

Se estableció la viabilidad del cultivo probiótico (Lactobacillus acidophilus) durante los 12 días de almacenamiento en refrigeración en cantidades $>1 \times 10^{6}$ ufc $/ \mathrm{ml}$ como lo indica la FAO.

Sensorialmente la bebida fermentada a base de arroz con probiótico presento aceptación en las características de color, sabor, olor, 
acidez, y textura, hasta el día 10 de

almacenamiento bajo refrigeración.

\section{REFERENCIAS BIBLIOGRÁFICAS}

Arci muhammet, et al., A. Lactic acid fermented cereal beverage as a traditional turkish food. 2002. Turquia.

Bautista J. selección de cepas nativas de Lactobacillus con actividad inhibitoria y tolerantes al etanol aisladas de masato. 2006. Lima, Perú.

Beltran, E., et al., Determinación de los parámetros fisicoquímicos y microbiológicos de la "chicha" producida en la localidad Santa Fe en la ciudad de Bogotá D.C. durante el primer semestre de 2006 [electrónico].

Duran, O. D., Trujillo, N. Y., Mejía, G. K. (2014). Capacidad de producción de alcohol de levaduras vinícolas sobre un sustrato a base de panela. @limentech, Ciencia y Tecnología Alimentaria. ISSN 1692-7125. Vol. 12 N¹. p. $78-85$.

Fula A. Angélica. (2010) Desarrollo de una bebida fermentada con adición de cocción de maíz. Bogotá: Universidad Nacional de Colombia. (2010) Facultad de Ciencias, Especialización en Ciencia y Tecnología de Alimentos, $33 \mathrm{p}$.
Giraffa, G. (2004). Studying the dynamics of microbial populations during food fermentation. FEMS Microbiol, Rev 28. p. 251-260.

Guerrero T., Juan. (2011). Utilización de probióticos (Lactobacillus plantarum) en la elaboración de una bebida de soya. Ecuador: Universidad técnica de Ambato, Facultad de Ciencias e Ingeniería en Alimentos, $157 \mathrm{p}$.

Guevara, J., Morales, R., Quintero, L. (2005). Evaluación de la utilización de probióticos en la fase de levante del ciclo de producción de la mojarra roja (Oreochromis sp.). Lima, Perú, p.25-26.

Instituto Colombiano de Normas Técnicas $Y$ Certificación. (1996). Bebidas alcohólicas. Cerveza. Bogotá D.C. ICONTEC, NTC 3854.

Instituto Colombiano de Normas Técnicas $Y$ Certificación. (2004) Manual de métodos analíticos para el control de calidad de bebidas alcohólicas. Bogotá D.C.ICONTEC, GTC 4. 
Instituto Colombiano de Normas Técnicas $Y$

Certificación. (2009). Microbiología de alimentos y productos para alimentación animal. Requisitos generales y directrices para análisis microbiológicos. Bogotá D.C.: ICONTEC, NTC 4092.

Ministerio de Salud y la Protección Social, Decreto 1686, (2012). Reglamento técnico sobre los requisitos sanitarios que se deben cumplir para fabricación de bebidas alcohólicas destinadas a consumo humano. Bogotá D.C.

Ramírez, G., Pedroza, J. (2001). Desarrollo de una Fermentación Alcohólica a pH Regulado y Temperatura de $25^{\circ} \mathrm{C}$ en el Biorreactor Bioflo 3000 m1227 y Estudio inicial de Fermentaciones en Sistema Continuo. Universidad de la Sabana Facultad de Ingeniería de Producción Agroindustrial. Bogotá, D.C.

Sancho, J., Bota, E. y Castro, JJ. (1999). Introducción al análisis sensorial de los alimentos. Ediciones Universitat de Barcelona. España, p. 142-143.

Sotero, V., García, D., Lessi, E. (1996). Bebida fermentada a partir de Pijuayo (Bactris Gasipaes H.B.K.) parámetros y evaluación. Folia amazonica, Vol. 8 (1). 